\title{
Conoscopic Measurement of Birefringence and Orientation in Biaxially Stretched Polymer Films and Sheets
}

\section{Brett L. Van Horn*,t and H. Henning Winter}

University of Massachusetts, Department of Chemical Engineering, Amherst, Massachusetts 01003

Received May 30, 2003; Revised Manuscript Received August 26, 2003

\begin{abstract}
The technique for using conoscopy to determine the optical orientation and birefringences of stretched polymer films is presented here in detail. The method is nondestructive and has the capacity for implementation both off-line and online during processing. Analysis was performed on films of polyethylene naphthalate (PEN) and poly(ethylene terephthalate) (PET), with birefringence results compared to measurements using a Berek compensator. The technique is highly effective at measuring the orientation and in-plane principal birefringence, though an accurate measure for a film's out-plane birefringence requires knowledge of at least an approximate value for the material's average refractive index.
\end{abstract}

\section{Introduction}

Biaxial stretching of many commercially important polymers, such as poly(ethylene terephthalate) (PET) and polyethylene naphthalate (PEN), is used to enhance the polymers' mechanical and optical properties. The stretching, carried out using various techniques including film blowing, blow molding, and sheet tentering, results in a molecular orientation of the polymer that gives rise to the anisotropy in physical and optical properties. There are many techniques frequently used to measure the molecular orientation of polymers including birefringence, $\mathrm{X}$-ray scattering, ultrasonic modulus measurements, FTIR spectroscopy, Raman spectroscopy, and NMR. The optical techniques using measurements of bi refringence or refractive indices are among the easiest to perform and also provide direct measurements of the optical anisotropy which is of primary importance in many applications.

Birefringence and refractive indices are most commonly measured using refractometry, retardation, or compensator techniques. Though these methods can provide detailed optical characterization of polymer films, they are either inappropriate for online installation or will only provide information along one direction of investigation. Frequently, bi refringence or refractive index measurements will be taken along the principal stretching directions parallel to film surface, the machine direction (MD) and the transverse direction (TD), and perhaps the direction normal to the film surface (ND). Quite often, however, the principal directions of the film's optical and molecular anisotropy are not collinear with the machine coordinate system, ${ }^{1-3}$ frequently resulting from the so-called "bowing" phenomena. It is therefore insufficient to measure the birefringences or refractive indices along MD, TD, and ND in an effort to optically characterize a polymer film. What is required, instead, is a method to quantify not only the magnitude of the optical anisotropy, either through birefringence or refractive index, but also the alignment of the optical anisotropy relative to the machine coordinate system.

* Corresponding author. E-mail: brett.van-horn@atofina.com.

† Current address: ATOFINA Chemicals, Inc., 900 First Ave., P.O. Box 61536, King of Prussia, PA 19406.
Conoscopy has long provided a powerful tool in the optical classification and investigation of crystals and minerals and the details of conoscopy may be found in many standard optical crystallography texts. ${ }^{4,5}$ Conoscopic investigation utilizes highly convergent polarized light to generate interference patterns that provide a wealth of detailed information that can be used to determine the orientation and magnitude of the optical anisotropy. These methods have been successfully applied to the analysis of biaxially stretched polymer films and have the capacity for being implemented online.

\section{Conoscopic Measurements}

In the conoscopic arrangement of a polarizing microscope, the conoscope, a condenser is inserted bel ow the sample to produce a cone of highly convergent light that is passed through the sample and brought into focus by the objective to form a real image. This image is not a magnification of the object itself but is instead a directions image, or interference figure, which is usually viewed by inserting a Bertrand-Amici lens between the upper polar (analyzer) and the ocular to focus on the back focal plane of the objective. The objective lens should be of high numerical aperture (NA) to maximize the angular spread of light rays imaged in the interference figure. Because the figure is a directions image, vertical displacements of the sample will not alter the observed interference figure so long as the material is reasonably homogeneous across the region probed by the cone of light. Positioning the center of the sample at the focal point of the convergent light will ensure that a minimum volume of material will be scanned. Alternatively, the interference figure may be projected to a screen for viewing by removing all of the microscope optics above the specimen except for the upper polar as shown in Figure 1.6

The benefits of this method over the conoscope for viewing interference figures is that there is no need to focus the image, that there are no delicate microscope optics above the sample, which may be useful for high temperature applications, and that the sample is not constrained to a narrow region between the condenser and the objective. The conoscope on the other hand has the advantages of well-defined optics that give rise to interference figures that may be more readily analyzed, 


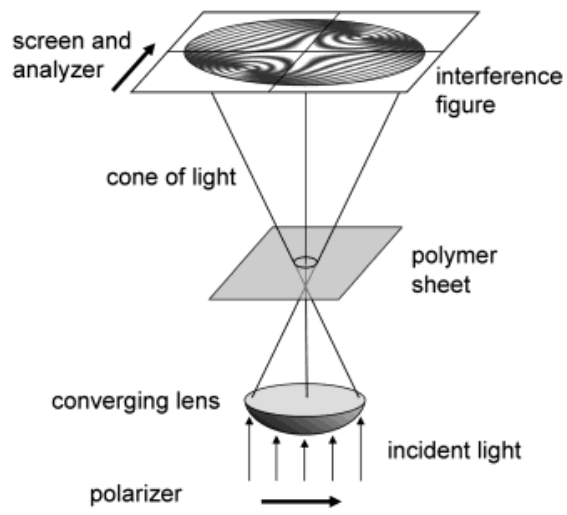

Figure 1. Schematic of conoscopic setup by projection of interference figure to screen.

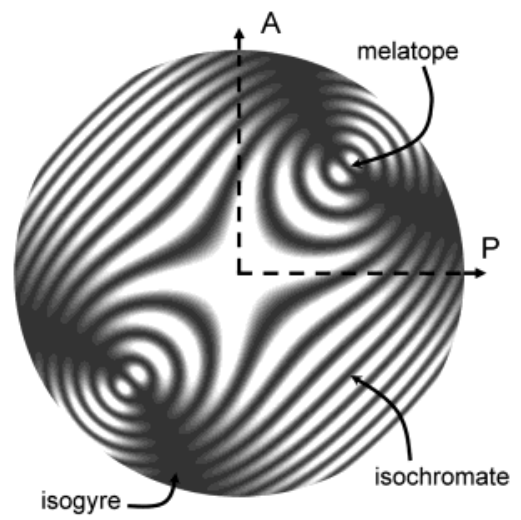

Figure 2. Example of biaxial interference figure.

where the edge of the field of view provides a built in reference point for measurements, and of the setup being fast and straightforward since polarizing microscopes are commercially availably. This work will only consider measurements from interference figures as seen through a conoscope, though the analysis would only be slightly al tered if the projection method were used.

Interference figures consist of two phenomena: isogyres and isochromates. The isogyres (see Figure 2), which may or may not be present, are dark curves resulting from extinction of light with vibration directions along the pol arizer and analyzer directions and are, therefore, sometimes referred to as curves of equal vibration direction.

The isochromates are lines of constant phase difference and with white light illumination they will be curves of constant color. Using monochromatic light illumination the isochromates will be curves of equal intensity. A melatope, or "eye", of the interference figure is a spot where the isogyres meet the isochromates of zero phase difference. Melatopes are characteristic points which mark the spot in the field of view for which light has traveled along the optic axis of the specimen and therefore emerges with zero phase difference. As shown by the example in Figure 3, the path difference, $d$, of the isochromatic lines of minimum intensity are integer multiples of the wavelength of light, $\lambda$, while the lines of maximum intensity are the odd-integer multiples of $\lambda$ halved.

The phase difference, $\delta$, is related to the path difference according to

$$
\delta=\mathrm{d} \frac{2 \pi}{\lambda}
$$

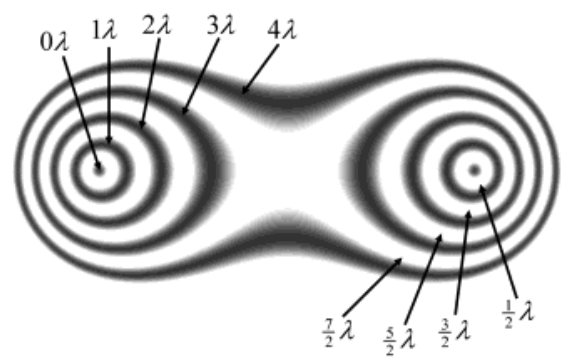

Figure 3. Path difference of isochromates as a function of wavel ength of light, $\lambda$.

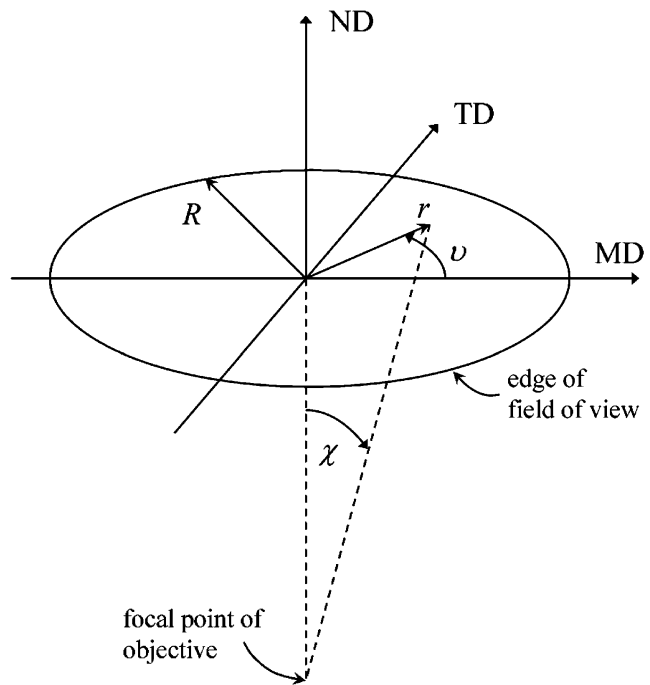

Figure 4. Definition of light path direction angles $\chi$ and $v$ with respect to the machine coordinate system. Refraction of light through sample is not shown.

The characteristics of interference figures depend on many factors, including birefringence, refractive index, orientation, $\lambda$, sample thickness, and optical setup. A careful analysis of conoscopic interference patterns will permit one to characterize the optical properties of the specimen. Since conoscopy probes the specimen over a range of wave vectors simultaneously, this analysis can be performed with minimal experiments.

A light ray reaching a given point of the interference figure will have made an angle of incidence $\chi$ with respect to the surface normal and an angle $v$ with respect to the fixed coordinate MD. The angle $v$ is measured directly from the interference figure according to Figure 4.

Since a conoscopic figure is approximately an orthographic projection of the interference phenomena, ${ }^{7} \chi$ can be calculated using the following equation:

$$
\frac{\mathrm{r}}{\mathrm{R}} \mathrm{NA}=\mathrm{n}_{0} \sin \chi
$$

where $r$ is the distance from the center of the field of view to the point of interest in the interference figure, $\mathrm{R}$ is the radius of the field of the view, $\mathrm{n}_{0}$ is the refractive index of the surrounding medium (air), and NA is the numerical aperture of the microscope objective. The data for our analysis will be of the form $\{\delta, \chi, v\}$, with points taken in the interference figure for known values of $\delta$ according to Figure 3 . The data will be fitted to the theoretical equation for the phase difference to determine the principal refractive indices of the polymer film. 


\section{Application of Technique}

The stretching of polymer sheets will lead to molecular orientation of the polymer chains parallel to the sheet surface. PET, PEN, and similar polymers containing flexibly linked, flat aromatic groups, like phenyl and naphthalate groups, will orient with their broad surfaces normal to the film surface. ${ }^{8}$ For these polymers, the intrinsic refractive index is greatest along the chain axis while the intrinsic refractive index normal to the aromatic groups is smallest. Stretching will then lead to an increase in the refractive index parallel to the film surface. Therefore, thelargest principal refractiveindex, $\mathrm{n}_{\mathrm{c}}$, of the refractive index ellipsoid (see Appendix A1) of stretched PEN and PET films will be parallel to the surface, with C rotated from MD by an angle $\phi$, and the smallest principal refractive index, $n_{a}$, will be normal to the surface, with A collinear with ND. When A is normal to the surface, the phase difference of monochromatic light of wavelength $\lambda$ transmitted through a homogeneous sheet of thickness $h$ is given by the following equation

$$
\delta=\frac{\pi \mathrm{h}}{\lambda\left(2 \epsilon_{\mathrm{a}}\right)^{1 / 2}}\left[(\Lambda 1+\Lambda 2)^{1 / 2}-(\Lambda 1-\Lambda 2)^{1 / 2}\right]
$$

where

$$
\begin{gathered}
\Lambda 1=4 \epsilon_{\mathrm{a}}\left(\epsilon_{\mathrm{b}}+\epsilon_{\mathrm{c}}\right)-2 \beta^{2}\left(2 \epsilon_{\mathrm{a}}+\epsilon_{\mathrm{b}}+\epsilon_{\mathrm{c}}\right)- \\
2\left(\epsilon_{\mathrm{b}}-\epsilon_{\mathrm{c}}\right) \beta^{2} \cos 2 \varphi \\
\begin{array}{c}
\Lambda 2=\left[16 \epsilon_{\mathrm{a}}\left(\epsilon_{\mathrm{a}}\left(\epsilon_{\mathrm{b}}-\epsilon_{\mathrm{c}}\right)^{2}-\beta^{2}\left(\epsilon_{\mathrm{b}}-\epsilon_{\mathrm{c}}\right)^{2}\right)+8 \beta^{2}\left(\epsilon_{\mathrm{b}}-\epsilon_{\mathrm{c}}\right) \times\right. \\
\left(\epsilon_{\mathrm{b}}+\epsilon_{\mathrm{c}}-2 \epsilon_{\mathrm{a}}\right)\left(\beta^{2}-2 \epsilon_{\mathrm{a}}\right) \cos 2 \varphi+2 \beta^{4}\left(8 \epsilon _ { \mathrm { a } } \left(\epsilon_{\mathrm{a}}-\epsilon_{\mathrm{b}}-\right.\right. \\
\left.\left.\left.\epsilon_{\mathrm{c}}\right)+2 \epsilon_{\mathrm{b}} \epsilon_{\mathrm{c}}+3\left(\epsilon_{\mathrm{b}}{ }^{2}+\epsilon_{\mathrm{c}}^{2}\right)\right)+2 \beta^{4}\left(\epsilon_{\mathrm{b}}-\epsilon_{\mathrm{c}}\right) \cos 4 \varphi\right]^{1 / 2} \\
\varphi=(5) \\
\beta=\mathrm{n}_{0} \sin \chi
\end{array}
\end{gathered}
$$

and $\epsilon_{\mathrm{a}}, \epsilon_{\mathrm{b}}$, and $\epsilon_{\mathrm{c}}$ are the principal dielectric tensor components which are the squares of the principal refractive indices $n_{a}, n_{b}$, and $n_{c}$ respectively. The derivation of eqs 3-5 is presented in Appendix A2. Though eq 3 may be theoretically sufficient to numerically fit "perfect" phase difference data in order to determine the principal refractive indices, it is insufficient experimentally. Even a small degree of error in experimental data may result in convergence to a set of incorrect or even theoretically impossible principal dielectric tensor components. To avoid this, one additional independent relationship among $\epsilon_{\mathrm{a}}, \epsilon_{\mathrm{b}}$, and $\epsilon_{\mathrm{c}}$ is required. This is principally done by specifying some form of average refractive index, or diel ectric component, which gives a relation as to the magnitude of the refractive indices as opposed to the difference between them. The sample average refractive index, $n_{a v g}$, is given by the average radius of the refractive index ellipsoid, which may be approximated as the geometric average of the three principal refractive indices

$$
\mathrm{n}_{\mathrm{avg}}=\left(\mathrm{n}_{\mathrm{a}} \mathrm{n}_{\mathrm{b}} \mathrm{n}_{\mathrm{c}}\right)^{1 / 3}
$$

which is equivalent to

$$
\epsilon_{\text {avg }}=\left(\epsilon_{\mathrm{a}} \epsilon_{\mathrm{b}} \epsilon_{\mathrm{c}}\right)^{1 / 3}
$$

provided $\mathrm{n}_{\text {avg }}{ }^{2}=\epsilon_{\text {avg. }}$. Rearrangement yields

$$
\epsilon_{\mathrm{c}}=\frac{\epsilon_{\mathrm{avg}}^{3}}{\epsilon_{\mathrm{a}} \epsilon_{\mathrm{b}}}
$$

Equation 10 is substituted into eqs 4 and 5 to reduce the number of fit parameters from three to two, where $\epsilon_{\text {avg }}$ is a known quantity. Since the birefringence is, in general, small compared to the refractive index, other types of averaging of the refractive indices may be used without significant loss accuracy. These include the arithmetic mean

$$
\mathrm{n}_{\mathrm{a} . \mathrm{m} .}=\frac{\mathrm{n}_{\mathrm{a}}+\mathrm{n}_{\mathrm{b}}+\mathrm{n}_{\mathrm{c}}}{3} \rightarrow \epsilon_{\mathrm{c}}={ }_{\left(3 \mathrm{n}_{\mathrm{am}}-\epsilon_{\mathrm{a}}^{1 / 2}-\epsilon_{\mathrm{b}}^{1 / 2}\right)^{2}}
$$

or the root-mean-squared

$$
\mathrm{n}_{\mathrm{rms}}=\left(\frac{\mathrm{n}_{\mathrm{a}}^{2}+\mathrm{n}_{\mathrm{b}}^{2}+\mathrm{n}_{\mathrm{c}}^{2}}{3}\right)^{1 / 2} \rightarrow \epsilon_{\mathrm{c}}=\begin{aligned}
& 3 \mathrm{n}_{\mathrm{rms}}{ }^{2}-\epsilon_{\mathrm{a}}-\epsilon_{\mathrm{b}}
\end{aligned}
$$

Similarly, since many studies measure only in-plane refractive indices, such as $\mathrm{n}_{M D}$ and $\mathrm{n}_{T D}$, an in-plane average refractive index relation may be used to provide the remaining independent equation.

Phase difference data as opposed to intensity profiles is used in the analysis. For starters, since intensity is measured in arbitrary units and dependent upon external factors such as the light source, exposure time, microscope optics, etc., intensity profiles would need to be rescaled so that the background intensity would match that of the model equations. Similarly, overexposed and underexposed images may lack the necessary details to fit intensity profile data to theoretical models. On the other hand, absolute values for the phase difference can be accurately determined from traces of the characteristic isochromates, defined in Figure 3, which are easily identified as the local relative extrema of the intensity surface. Data points are selected from the interference figure al ong traces of the isochromates for at least two independent values of $\varphi$, such as along the $\mathrm{B}$ and $\mathrm{C}$ axes projections for $\varphi=\pi / 2$ and $\varphi=0$, respectively. These data will then be fit to eq 3 using standard nonlinear regression with known parameters $\mathrm{h}, \lambda, \mathrm{n}_{\mathrm{avg}}$, and $\mathrm{n}_{0}$ and fit parameters $\epsilon_{\mathrm{a}}$ and $\epsilon_{\mathrm{b}} . \epsilon_{\mathrm{c}}$ is then calculated using eq 10 to yield the complete set of principal dielectric tensor components, which in turn provide the refractive indices.

The situation will be slightly complicated if the melatopes are not visible because the actual phase order of the isochromates will not be known. However, the difference in phase order between the isochromates is still known relative to an integer offset, 0 , which is a fit parameter. The phase difference equation to fit the data then becomes

$\delta=\frac{\pi \mathrm{h}}{\lambda\left(2 \epsilon_{\mathrm{a}}\right)^{1 / 2}}\left[(\Lambda 1+\Lambda 2)^{1 / 2}-(\Lambda 1-\Lambda 2)^{1 / 2}\right]+\frac{2 \pi \mathrm{o}}{\lambda}$

If the nonlinear regression procedure does not allow for integer fit parameters then the solve routine is performed twice: The first fit converges on the solution with the best fit value for 0 . This value is rounded to the nearest integer which then becomes a known 
parameter for the second fit to find the values for the principal refractive indices.

Iterative fit routines require initial guess values of the fit parameters and a poor choice of these initial values may result in convergence to a nonoptimal or unacceptable set of parameters. In this study, Mathematica 3.0, by Wolfram Research, Inc., was used to fit the data to the model through nonlinear regression. Mathematica's built-in function F indMinimum was used as the optimization method to minimize the sum of the squared residuals. This method was highly insensitive to the choice of the initial guess values so long as they were within a reasonable range, such as $1<\epsilon_{\mathrm{a}}<\epsilon_{\mathrm{b}}<$ 5. However, when a Levenberg-Marquardt method was used instead, the fit would only converge to the optimal solution within a narrow range of guess values, though this problem may be circumvented by performing the fit several times while varying the guess values through a marching procedure in search of the optimal fit.

If the melatopes are visible in the interference figure, an alternative approach may be used to solve for $\epsilon_{\mathrm{a}}, \epsilon_{\mathrm{b}}$, and $\epsilon_{\mathrm{c}}$. We are required three equations to sol ve for the three unknowns, and these may be obtained by considering the optical properties in terms of the material's average refractive index, birefringence, and biaxiallity. The average refractive index, which addresses the magnitude of the refractive indices, has al ready been defined through eq 9. An equation primarily addressing the birefringence is obtained from eq 3 , using a single data point taken from a non-zero-order extinction isochromate. The biaxiallity is a measure of the difference between the principal birefringences $\Delta n_{c-b}=\left(n_{c}-n_{b}\right)$ and $\Delta n_{b-a}=\left(n_{b}-n_{a}\right)$. For a uniaxial material the appropriate principal birefringence is simply set equal to zero. F or biaxial materials, the biaxiallity is expressed through the optic angle (see Appendix A1), which is defined in terms of the refractive indices according to either eq 16 or 17 . When the A-axis is normal to the film surface we can combine eq 17 with Snell's law of refraction to yield

$$
\mathrm{n}_{0} \sin \chi_{\text {melatope }}=\sqrt{\epsilon_{\mathrm{b}}} \sin \left(\cos ^{-1} \sqrt{\frac{\epsilon_{\mathrm{c}}\left(\epsilon_{\mathrm{b}}-\epsilon_{\mathrm{a}}\right)}{\epsilon_{\mathrm{b}}\left(\epsilon_{\mathrm{c}}-\epsilon_{\mathrm{a}}\right)}}\right)
$$

where $\chi$ melatope is the angle of incidence for light traveling along an optic axis and is measured from the position of a melatope in the interference figure using eq 2 . Since the melatopes lie along a line of $\varphi=0$, the data point chosen for insertion into eq 3 is best taken along $\varphi=$ $\pi / 2$, or close to it, to hel $p$ capture the curvature of the isochromates. This principle applies to the acquisition of the overall data in general: data points should be selected from a range of $\varphi$, particularly around $\varphi=0$ and $\pi / 2$, and not biased toward any one direction.

\section{Experimental Results and Discussion}

Two biaxially stretched, industrial films, one PE N and one PET, were studied using the procedure described above. The stretching ratios and processing conditions of the films were not provided by the film supplier. The PEN film is $90 \mu \mathrm{m}$ thick and the PET film is $101 \mu \mathrm{m}$ thick. A Nikon Eclipse e400 polarizing microscope, equipped with a 0.80 numerical aperture objective lens and a band-pass filter with peak transmittance at 445.0 $\mathrm{nm}$ and a bandwidth of $50.0 \mathrm{~nm}$, was used to obtain the conoscopic interference figures for analysis. Samples were taken from films at two positions, 1 and 2, which a)

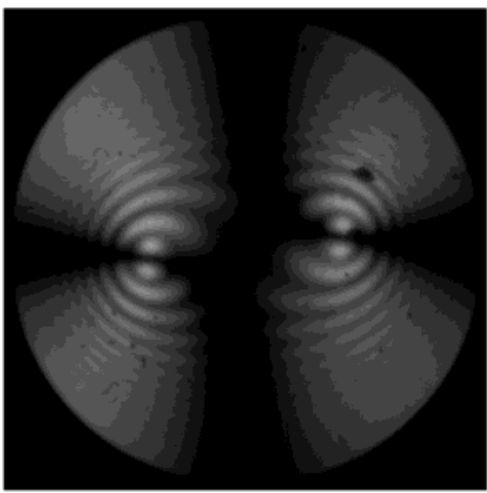

b)

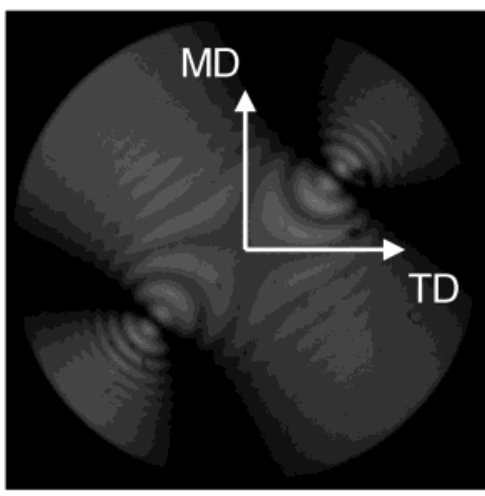

Figure 5. Interference figures for PEN: (a) PEN-1 and (b) PEN-2. NA $=0.80, \lambda=445.0 \mathrm{~nm}$.

a)

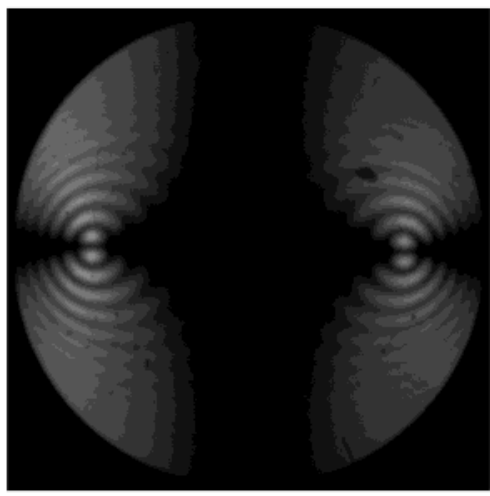

b)

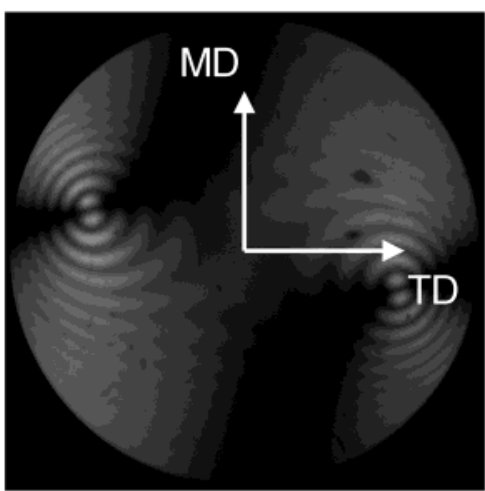

Figure 6. Interference figures for PET: (a) PET-1 and (b) PET-2. NA $=0.80, \lambda=445.0 \mathrm{~nm}$.

were $20 \mathrm{~cm}$ apart along TD and at the same value of MD in each case. Figures 5 and 6 show the interference figures for the PEN and PET films, respectively.

The optical properties for the film samples are given in Tables 1 and 2. The films are clearly biaxial, showing two distinct melatopes, with the A-axis normal to the film surface $\left(\theta=\pi / 2\left(90^{\circ}\right)\right.$ and $\left.\psi=\pi / 2\left(90^{\circ}\right)\right)$. This was 
Table 1. Optical Properties for PEN Samples

conoscopy

\begin{tabular}{|c|c|c|c|c|c|c|c|c|}
\hline \multirow[b]{2}{*}{ position } & & \multirow[b]{2}{*}{$\begin{array}{l}\text { Berek compensator } \\
\qquad \Delta n_{c-b}\end{array}$} \\
\hline & $\phi$, deg & $\mathrm{n}_{\text {avg }}$ & $\mathrm{n}_{\mathrm{a}}$ & $\mathrm{n}_{\mathrm{b}}$ & $\mathrm{n}_{\mathrm{c}}$ & $\Delta \mathrm{n}_{\mathrm{b}-\mathrm{a}}$ & $\Delta \mathrm{n}_{\mathrm{c}-\mathrm{b}}$ & \\
\hline \multirow[t]{3}{*}{1} & 7 & 1.64 & 1.4950 & 1.7127 & 1.7227 & 0.2177 & 0.0101 & 0.0144 \\
\hline & & 1.77 & 1.6018 & 1.8556 & 1.8656 & 0.2537 & 0.0101 & \\
\hline & & 1.90 & 1.7073 & 1.9993 & 2.0094 & 0.2920 & 0.0101 & \\
\hline \multirow[t]{3}{*}{2} & 39 & 1.64 & 1.4928 & 1.7113 & 1.7265 & 0.2185 & 0.0152 & 0.0207 \\
\hline & & 1.77 & 1.5991 & 1.8546 & 1.8698 & 0.2555 & 0.0152 & \\
\hline & & 1.90 & 1.7040 & 1.9987 & 2.0139 & 0.2947 & 0.0152 & \\
\hline
\end{tabular}

Table 2. Optical Properties for PET Samples

conoscopy

\begin{tabular}{|c|c|c|c|c|c|c|c|c|}
\hline position & $\phi$, deg & $\mathrm{n}_{\text {avg }}$ & $\mathrm{n}_{\mathrm{a}}$ & $\mathrm{n}_{\mathrm{b}}$ & $\mathrm{n}_{\mathrm{c}}$ & $\Delta \mathrm{n}_{\mathrm{b}-\mathrm{a}}$ & $\Delta \mathrm{n}_{\mathrm{c}-\mathrm{b}}$ & $\begin{array}{c}\text { Berek compensator } \\
\qquad \Delta \mathrm{n}_{\mathrm{c}-\mathrm{b}}\end{array}$ \\
\hline \multirow[t]{3}{*}{1} & 1 & 1.57 & 1.4777 & 1.6092 & 1.6274 & 0.1315 & 0.0182 & 0.0228 \\
\hline & & 1.60 & 1.5039 & 1.6412 & 1.6594 & 0.1373 & 0.0182 & \\
\hline & & 1.66 & 1.5562 & 1.7054 & 1.7236 & 0.1492 & 0.0182 & \\
\hline \multirow[t]{3}{*}{2} & 14 & 1.57 & 1.4797 & 1.6078 & 1.6267 & 0.1281 & 0.0189 & 0.0234 \\
\hline & & 1.60 & 1.5060 & 1.6397 & 1.6586 & 0.1337 & 0.0189 & \\
\hline & & 1.66 & 1.5585 & 1.7038 & 1.227 & 0.1453 & 0.0189 & \\
\hline
\end{tabular}

verified according to the procedure in Appendix A3 by observing the change in position of the isochromates after the insertion of a quartz wedge into the optical path. $\phi$ was measured as the angle between MD and the line perpendicular to a line connecting the two melatopes. Interference data points were taken from each figure at the two melatopes and at five random locations along each isochromate up to fifth order extinction. Data were not taken where the isochromates were hidden by the isogyres. The data were fit to the mathematical model over a range of values for the average refractive index. From literature, the average refractive index for PET is usually in the range of 1.571.66. 2,9-13 Much less is reported on the properties of PEN, but the range of the average refractive index is reported to be $1.64-1.90 .{ }^{14,15}$ From the fit results, the in-plane birefringence $\Delta \mathrm{n}_{\mathrm{c}-\mathrm{b}}$ and the out-plane birefringence $\Delta n_{b-a}$ were calculated. Measurements of in-plane birefringence were also taken using an Olympus U-CTB Berek compensator.

The optical orientation is aligned closely with the machine frame of reference for samples taken in position 1 (PEN-1 and PET-1), where for PEN $\phi=7^{\circ}$ and for PET $\phi=1^{\circ}$. However, there is a significant deviation for samples taken from position 2 (PEN-2 and PET-2), where for PEN $\phi=39^{\circ}$ and for PET $\phi=14^{\circ}$. The calcul ated birefringence using conoscopy agrees reasonably well with the values measured using the Berek compensator. In all cases the in-plane birefringences measured conoscopically were approximately 0.005 less than the compensator measurements, though this may have been due to miscalibration of the compensator. Of particular significance is that the calculated in-plane birefringence, $\Delta n_{c-b}$, is almost completely insensitive to the average refractive index, $n_{a v g}$, used for the numerical fit routine within the ranges studied. The out-plane birefringence, $\Delta n_{b-a}$, however, shows a more significant dependence on the choice of $n_{\text {avg. }}$. The ranges for the refractive index studied are quite broad, however, especially for PEN, so a closer approximation of the average refractive index for an individual study will greatly improve the precision of the calculated out-plane birefringence.

Though not shown here, the orientation and birefringence are nearly independent of MD for both films. In contrast, they were highly dependent upon TD, and

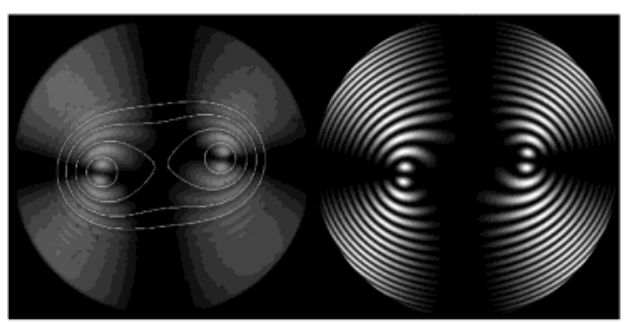

a)

b)

Figure 7. PEN-1: (a) experimental interference figure with overlay of calculated extinction isochromates (white lines) up to fifth-order extinction; (b) simulated interference figure.

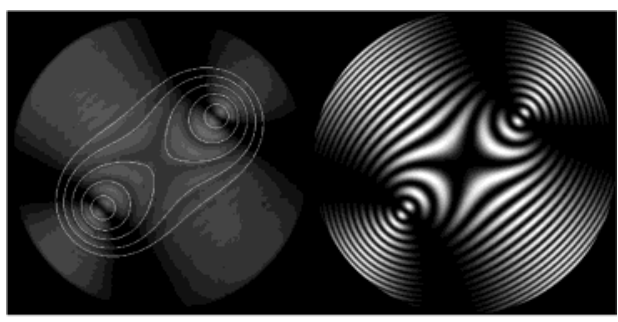

a)

b)

Figure 8. PEN-2: (a) experimental interference figure with overlay of calculated extinction isochromates (white lines) up to fifth order extinction; (b) simulated interference figure.

were seen to vary smoothing from position 1 to position 2 , becoming increasingly off-axis with increasing birefringence. Similar results have been reported by other researches for biaxially stretched films. ${ }^{2,3,16}$ The usual source of this TD dependence on the properties of films biaxially stretched in a tenter process is the bowing phenomena, ${ }^{16,17}$ the cause of which is believed to be machine direction forces arising from the transverse stretching by the tenter and heat shrinkage. ${ }^{18}$

To demonstrate the quality of the fit results, the positions of the extinction isochromates, up to fifth-order extinction, were calculated via eqs 2 and 24 . The calculations were performed using the fit results for PEN with $n_{\text {avg }}=1.77$ and for PET with $n_{\text {avg }}=1.60$ as given in Tables 1 and 2 . The calculated extinction isochromates have been overlaid onto the experimental interference figures in Figures 7-10. Also shown are simulated interference figures with the phase difference calculated for the same set of optical parameters with 


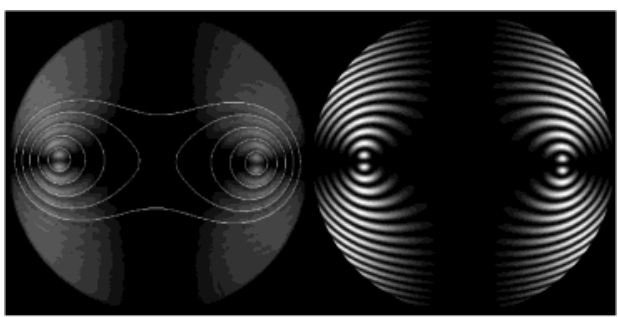

a)

b)

Figure 9. PET-1: (a) experimental interference figure with overlay of calculated extinction isochromates (white lines) up to fifth order extinction; (b) simulated interference figure.

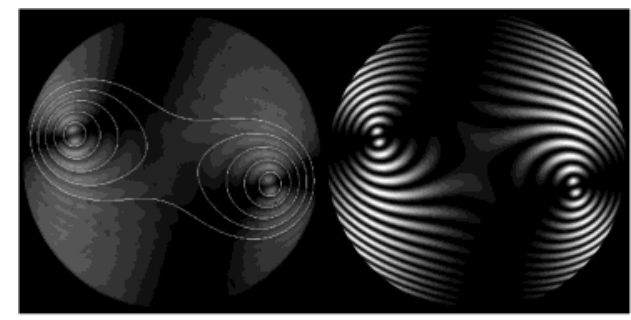

a)

b)

Figure 10. PET-2: (a) experimental interference figure with overlay of calculated extinction isochromates (white lines) up to fifth order extinction; (b) simulated interference figure.

the phase difference calculated using eq 24 while the vibration directions and intensity were calculated according to the definitions given in ref 19 for biaxial crystals.

The purpose of this work was not to analyze particular film samples but to present a technique for the analysis of stretched polymer films in general. Therefore, a detailed study to fully characterize the optical properties across the samples was not undertaken. This is also why no effort was made to obtain films with known processing conditions, such as draw ratio and temperature history, for which meaningful comparisons between optical properties and processing history could be made.

This study was performed off-line, with measurements taken manually. With the current state of image analysis software packages, it should be possible to automate the analysis procedure so that this technique may be implemented on-line in a film-tentering process for either process control or quality control purposes. Using a very narrow band-pass filter or a true monochromatic light source, such a laser, will sharpen the interference figures, which will aid the image analysis and pattern recognition, thereby facilitating the automation of the process. Also, viewing the projected interference figures directly, as opposed to through the microscope as mentioned earlier, is perhaps more applicable to industrial applications since focusing of the interference figures and careful positioning of the microscope optics will not be required.

The conoscopic figures for PEN and PET showed several orders of extinction of the isochromates, which aided the determination of birefringence. In order for the calculation of birefringence using this conoscopic method the phase difference for the transmitted light needs to be high enough so that at least the first-order extinction isochromate is clearly distinguishable, though several visible extinction isochromates are preferred. A decrease in the film thickness will result in a corre sponding decrease in phase difference, so that for thin films there may not be a sufficient order of extinction to calculate the birefringence. Decreasing the birefringence will also decrease the phase difference, so that films with low birefringence may have few if any isochromates. With PEN and PET, low birefringence would result from low molecular order. I sotactic polypropylene (iPP), however, has an intrinsic birefringence around an order of magnitude less than that of PEN or PET, ${ }^{20,21}$ so that for even a highly ordered iPP film the overall birefringence will still below. The situation with iPP is further complicated because under some stretching conditions the contributions to the overall birefringence from the crystalline and amorphous regions will counteract, resulting in low or zero birefringence under certain circumstances. ${ }^{21}$ Therefore, for thin films, films with low molecular order, or films from polymers with low intrinsic birefringence this technique may not be able to determine the film birefringence. The orientation, however, might still be determined from the appearance and position of the isogyres. The opposite situation, that of too many isochromates to easily distinguish between them, can be overcome by simply using a low power objective lens, thereby decreasing the range of angles of incidence viewed in the conoscopic figure and thus decreasing the number of isochromates.

\section{Conclusions}

Conoscopy provides an easy and inexpensive means of measuring the optical orientation and birefringence of stretched polymer films. The orientation and, provided the film thickness is known, in-plane birefringence can be accurately determined without intimate knowledge of the material's average refractive index, though an accurate determination of the out-plane birefringence does require at least an approximate value. This technique is nondestructive and has the potential for being implemented on-line during processing. Like many other birefringent techniques, conoscopy is sample averaging, where the measured birefringence is averaged over the light path and includes components from the amorphous and crystalline phases as well as form birefringence. The principal in-plane birefringences determined in this study for the PEN and PET samples agree with measurements taken using a Berek compensator. The TD dependence on the orientation is also clearly seen and easily measured using conoscopy, making conoscopy an effective tool for monitoring "bowing" phenomena or other sources of off-axis optical orientation.

\section{Appendixes}

A1. Refractive Index Ellipsoid. The variation of the refractive index as a function of the vibration direction of light is described with the refractive index ellipsoid, or indicatrix, where the principal radi i of the indicatrix are equal to the three principal refractive indices $n_{a}, n_{b}$, and $n_{c}$ as shown in Figure 11

The refractive index for a given vibration direction is equal to the distance from the center to the surface of the ellipsoid defined according to

$$
\frac{A^{2}}{n_{a}^{2}}+\frac{B^{2}}{n_{b}^{2}}+\frac{C^{2}}{n_{c}^{2}}=1
$$

For isotropic materials the three principal refractive indices are equal, for uniaxial materials two are equal, and for biaxial materials none are equal. Light with an arbitrary wave normal direction will be normal to an 


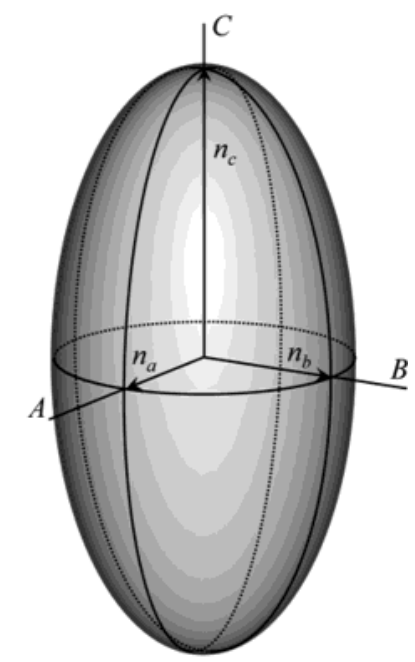

Figure 11. Refractive index ellipsoid (Indicatrix).

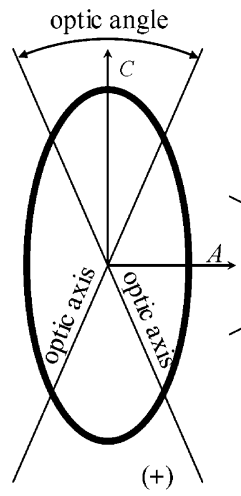

a)

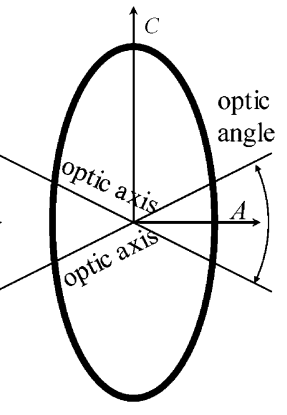

$(-)$ b)

Figure 12. Behavior of optic axes and optic angle for a material that is a) biaxially positive and (b) biaxially negative.

elliptical cross section of the indicatrix and the only two vibration directions associated with this wave normal are along the semimajor and semiminor axes of the elliptical cross section. The refractive indices for these vibration directions are thereby given as the length of the semimajor and semiminor axes. The cross section normal to an optic axis is circular; therefore the material will appear isotropic for light with a wave normal along the optic axis. Uniaxial materials have one optic axis while biaxial materials have two.

We define the refractive indices such that $n_{a} \leq n_{b} \leq$ $\mathrm{n}_{\mathrm{c}}$. If the material is isotropic then $\mathrm{n}_{\mathrm{a}}=\mathrm{n}_{\mathrm{b}}=\mathrm{n}_{\mathrm{c}}$. There are two possibilities for uniaxial materials. If $n_{a}=n_{b}<$ $\mathrm{n}_{\mathrm{c}}$, the material is said to be optically positive, where the indicatrix is prolate and $C$ is the optic axis. If $n_{a}<$ $\mathrm{n}_{\mathrm{b}}=\mathrm{n}_{\mathrm{c}}$, the material is optically negative, where the indicatrix is oblate and $A$ the optic axis. With biaxial materials $\mathrm{n}_{\mathrm{a}}<\mathrm{n}_{\mathrm{b}}<\mathrm{n}_{\mathrm{c}}$ and the two optic axes will lie in the $A-C$ plane (see Figure 12).

The angle between the optic axes bisected by $C$ is $2 V_{C}$ and the angle between the two optic axes bisected by $A$ is $2 V_{A}$. The acute angle between the optic axes is called the optic angle, $2 \mathrm{~V}$, and the coordinate which bisects the optic angle is called the acute bisectrix. For positive biaxial materials $\mathrm{C}$ is the acute bisectrix $\left(2 \mathrm{~V}=2 \mathrm{~V}_{\mathrm{C}}\right)$, where $\left(n_{c}-n_{b}\right)<\left(n_{b}-n_{a}\right)$, while for negative biaxial materials $A$ is the acute bisectrix $\left(2 \mathrm{~V}=2 \mathrm{~V}_{\mathrm{A}}\right)$, where $\left(\mathrm{n}_{\mathrm{b}}\right.$ $\left.-n_{a}\right)<\left(n_{c}-n_{b}\right)$. The relationships between the $V_{A}$ and $V_{C}$ with the principal refractive indices are shown below: ${ }^{4,5}$

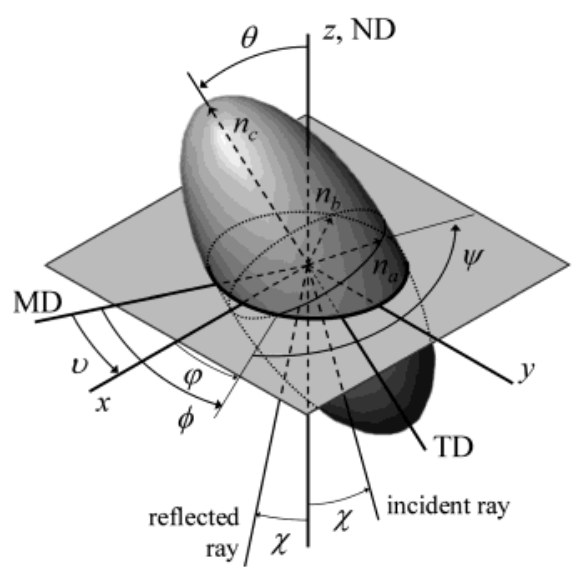

Figure 13. Machine frame of reference, refractive index tensor, and incident ray coordinate systems. Euler angles of dielectric tensor $\theta, \varphi$, and $\psi$ with respect to the $\mathrm{x}, \mathrm{y}, \mathrm{z}$ coordinate system. $\mathrm{x}-\mathrm{y}$ rotated from MD-TD by angle $v$ (figure adapted from ref 22).

$$
\begin{gathered}
\cos ^{2} V_{A}=\frac{n_{C}^{2}\left(n_{B}^{2}-n_{A}^{2}\right)}{n_{B}^{2}\left(n_{C}^{2}-n_{A}^{2}\right)} \\
\cos ^{2} V_{C}=\frac{n_{A}^{2}\left(n_{C}+n_{B}\right)\left(n_{C}-n_{B}\right)}{n_{B}^{2}\left(n_{C}+n_{A}\right)\left(n_{C}-n_{A}\right)}
\end{gathered}
$$

A2. Phase Difference Determination. The interference phenomena of conoscopic figures can be pre dicted using the $4 \times 4$ matrix method for finding reflection and transmission by anisotropic planar structures with obliquely or normally incident light.22 The coordinate systems for this analysis are shown in Figure 13.

The machine frame of reference is defined by the machine and transverse directions MD and TD, respectively. The refractive index tensor is defined by the refractive index ellipsoid coordinates $A, B$, and $C$. The incident light coordinate system axes are $\mathrm{x}, \mathrm{y}$, and $\mathrm{z}$. Light with a complex time dependence exp(i $\omega$ t), coming from a surrounding medium with refractive index $n_{0}$, is incident on the sample by an angle $\chi$ with respect to the z-axis, the surface normal. From Maxwell's equations the linear differential equations for the tangential components of the electric and magnetic field vectors are

$$
\frac{\mathrm{d} \psi}{\mathrm{d} z}=-\frac{\mathrm{i} \omega}{\mathrm{c}} \Delta(\mathrm{z}) \psi
$$

where $c$ is the speed of light in a vacuum, $\Delta$ is a $4 \times 4$ matrix, and

$$
\psi=\left(\mathrm{E}_{\mathrm{x}}, \mathrm{H}_{\mathrm{y}}, \mathrm{E}_{\mathrm{y}},-\mathrm{H}_{\mathrm{x}}\right)^{\top}
$$

For an anisotropic dielectric medium with principal refractive indices $n_{a}, n_{b}$, and $n_{c}$ of the refractive index ellipsoid and aligned with subtending Euler angles $\theta$, $\varphi$, and $\psi$ with respect to the $\mathrm{x}, \mathrm{y}, \mathrm{z}$ coordinate system, the $4 \times 4$ differential matrix will be

$$
\Delta=\left[\begin{array}{llll}
\Delta_{11} & \Delta_{12} & \Delta_{13} & 0 \\
\Delta_{21} & \Delta_{11} & \Delta_{23} & 0 \\
0 & 0 & 0 & \Delta_{34} \\
\Delta_{23} & \Delta_{13} & \Delta_{43} & 0
\end{array}\right]
$$


where

$$
\begin{aligned}
& \Delta_{11}=-\beta\left(\frac{\epsilon_{13} \cos \varphi-\epsilon_{23} \sin \varphi}{\epsilon_{33}}\right) \\
& \Delta_{12}=1-\frac{\beta^{2}}{\epsilon_{33}} \\
& \Delta_{13}=-\beta\left(\frac{\epsilon_{13} \sin \varphi+\epsilon_{23} \cos \varphi}{\epsilon_{33}}\right) \\
& \Delta_{21}=\left[\left(\frac{\epsilon_{11}-\epsilon_{22}}{2}-\frac{\epsilon_{13}^{2}-\epsilon_{23}^{2}}{2 \epsilon_{33}}\right) \cos 2 \varphi-\right. \\
& \left.\left(\epsilon_{12}-\frac{\epsilon_{13} \epsilon_{23}}{\epsilon_{33}}\right) \sin 2 \varphi\right]+\left(\frac{\epsilon_{11}+\epsilon_{22}}{2}-\frac{\epsilon_{13}{ }^{2}+\epsilon_{23}{ }^{2}}{2 \epsilon_{33}}\right) \\
& \Delta_{23}=\left[\left(\frac{\epsilon_{11}-\epsilon_{22}}{2}-\frac{\epsilon_{13}{ }^{2}-\epsilon_{23}{ }^{2}}{2 \epsilon_{33}}\right) \sin 2 \varphi+\right. \\
& \left.\left(\epsilon_{12}-\frac{\epsilon_{13} \epsilon_{23}}{\epsilon_{33}}\right) \cos 2 \varphi\right] \\
& \Delta_{34}=1 \\
& \Delta_{43}=-\left[\left(\frac{\epsilon_{11}-\epsilon_{22}}{2}-\frac{\epsilon_{13}{ }^{2}-\epsilon_{23}{ }^{2}}{2 \epsilon_{33}}\right) \cos 2 \varphi-\right. \\
& \left.\left(\epsilon_{12}-\frac{\epsilon_{13} \epsilon_{23}}{\epsilon_{33}}\right) \sin 2 \varphi\right]+\left(\frac{\epsilon_{11}+\epsilon_{22}}{2}-\frac{\epsilon_{13}{ }^{2}+\epsilon_{23}{ }^{2}}{2 \epsilon_{33}}\right)-\beta^{2}
\end{aligned}
$$

and

$$
\begin{gathered}
\epsilon_{11}=\epsilon_{\mathrm{a}} \cos ^{2} \psi+\epsilon_{\mathrm{b}} \sin ^{2} \psi \\
\epsilon_{12}=\left(\epsilon_{\mathrm{a}}-\epsilon_{\mathrm{b}}\right) \sin \psi \cos \psi \cos \theta \\
\epsilon_{13}=\left(\epsilon_{\mathrm{a}}-\epsilon_{\mathrm{b}}\right) \sin \psi \cos \psi \sin \theta \\
\epsilon_{22}=\left(\epsilon_{\mathrm{a}} \sin ^{2} \psi+\epsilon_{\mathrm{b}} \cos ^{2} \psi\right) \cos ^{2} \theta+\epsilon_{\mathrm{c}} \sin ^{2} \theta \\
\epsilon_{23}=\left(\epsilon_{\mathrm{a}} \sin ^{2} \psi+\epsilon_{\mathrm{b}} \cos ^{2} \psi-\epsilon_{\mathrm{c}}\right) \sin \theta \cos \theta \\
\epsilon_{33}=\left(\epsilon_{\mathrm{a}} \sin ^{2} \psi+\epsilon_{\mathrm{b}} \cos ^{2} \psi\right) \sin ^{2} \theta+\epsilon_{\mathrm{c}} \cos ^{2} \theta \\
\beta=\mathrm{n}_{0} \sin \chi
\end{gathered}
$$

and the principal dielectric tensor components are the squares of the principal refractive indices

$$
\begin{aligned}
\epsilon_{\mathrm{a}} & =\mathrm{n}_{\mathrm{a}}{ }^{2} \\
\epsilon_{\mathrm{b}} & =\mathrm{n}_{\mathrm{b}}{ }^{2} \\
\epsilon_{\mathrm{c}} & =\mathrm{n}_{\mathrm{c}}{ }^{2}
\end{aligned}
$$

The matrix $\Delta$ has four eigenvalues, $\lambda_{1-4}$, corresponding to the $z$ components of the forward- and backwardpropagating waves.

F or a homogeneous anisotropic medium of thickness $\mathrm{h}$, the phase difference, $\delta$, of the forward-propagating transmitted waves is given by

$$
\delta=\frac{2 \pi \mathrm{h}}{\lambda}\left(\lambda_{3}-\lambda_{1}\right)
$$

where $\lambda$ is the wavelength of light. For uniaxial materi- als the angle $\psi$ is arbitrary and general expressions for the eigenvalues of $\Delta$ under these conditions have already been derived. ${ }^{23} \mathrm{~F}$ or the more general case of biaxial materials expressions for the eigenvalues are not as forthcoming, and only approximate solutions for specific orientations could be found in the literature. 4,19 However, when one of the principal axes of the biaxial indicatrix is collinear with ND then general expressions for the eigenvalues are obtainable. The expressions are written in terms of the following two functions of the dielectric tensor components, where the subscripts $i, j$, and $\mathrm{k}$ represent the three principal dielectric constant subscripts $a, b$, and $c$ :

$$
\begin{aligned}
& \Lambda 1_{\mathrm{i}, \mathrm{j}, \mathrm{k}}=4 \epsilon_{\mathrm{i}}\left(\epsilon_{\mathrm{j}}+\epsilon_{\mathrm{k}}\right)-2 \beta^{2}\left(2 \epsilon_{\mathrm{i}}+\epsilon_{\mathrm{j}}+\epsilon_{\mathrm{k}}\right)- \\
& 2\left(\epsilon_{\mathrm{j}}-\epsilon_{\mathrm{k}}\right) \beta^{2} \cos 2 \varphi \\
& \Lambda 2_{\mathrm{i}, \mathrm{j}, \mathrm{k}}=\left[16 \epsilon_{\mathrm{i}}\left(\epsilon_{\mathrm{i}}\left(\epsilon_{\mathrm{j}}-\epsilon_{\mathrm{k}}\right)^{2}-\beta^{2}\left(\epsilon_{\mathrm{j}}-\epsilon_{\mathrm{k}}\right)^{2}\right)+\right. \\
& 8 \beta^{2}\left(\epsilon_{\mathrm{j}}-\epsilon_{\mathrm{k}}\right)\left(\epsilon_{\mathrm{j}}+\epsilon_{\mathrm{k}}-2 \epsilon_{\mathrm{i}}\right)\left(\beta^{2}-2 \epsilon_{\mathrm{i}}\right) \cos 2 \varphi+ \\
& 2 \beta^{4}\left(8 \epsilon_{\mathrm{i}}\left(\epsilon_{\mathrm{i}}-\epsilon_{\mathrm{j}}-\epsilon_{\mathrm{k}}\right)+2 \epsilon_{\mathrm{j}} \epsilon_{\mathrm{k}}+3\left(\epsilon_{\mathrm{j}}^{2}+\epsilon_{\mathrm{k}}{ }^{2}\right)\right)+ \\
& \left.2 \beta^{4}\left(\epsilon_{\mathrm{j}}-\epsilon_{\mathrm{k}}\right) \cos 4 \varphi\right]^{1 / 2}
\end{aligned}
$$

The phase difference for each of the cases where a principal biaxial indicatrix axis is collinear with ND can then be written as follows.

I. A normal to the surface $(\theta=\pi / 2, \psi=\pi / 2)$ :

$$
\begin{aligned}
& \delta=\frac{\pi \mathrm{h}}{\lambda\left(2 \epsilon_{\mathrm{a}}\right)^{1 / 2}}\left[\left(\Lambda 1_{\mathrm{a}, \mathrm{b}, \mathrm{c}}+\Lambda 2_{\mathrm{a}, \mathrm{b}, \mathrm{c}}\right)^{1 / 2}-\right. \\
&\left.\left(\Lambda 1_{\mathrm{a}, \mathrm{b}, \mathrm{c}}-\Lambda 2_{\mathrm{a}, \mathrm{b}, \mathrm{c}}\right)^{1 / 2}\right]
\end{aligned}
$$

II. B normal to the surface $(\theta=\pi / 2, \psi=0)$ :

$$
\begin{aligned}
& \delta=\frac{\pi h}{\lambda\left(2 \epsilon_{\mathrm{b}}\right)^{1 / 2}}\left[\left(\Lambda 1_{\mathrm{b}, \mathrm{a}, \mathrm{c}}+\Lambda 2_{\mathrm{b}, \mathrm{a}, \mathrm{c}}\right)^{1 / 2}-\right. \\
&\left.\left(\Lambda 1_{\mathrm{b}, \mathrm{a}, \mathrm{c}}-\Lambda 2_{\mathrm{b}, \mathrm{a}, \mathrm{c}}\right)^{1 / 2}\right]
\end{aligned}
$$

III. C normal to the surface $(\theta=0, \psi=\pi / 2)$ :

$$
\begin{array}{r}
\delta=\frac{\pi h}{\lambda\left(2 \epsilon_{c}\right)^{1 / 2}}\left[\left(\Lambda 1_{c, b, a}+\Lambda 2_{c, b, a}\right)^{1 / 2}-\right. \\
\left.\left(\Lambda 1_{c, b, a}-\Lambda 2_{c, b, a}\right)^{1 / 2}\right]
\end{array}
$$

When a biaxial specimen is not oriented along one of these three principal cases it may still be possible to obtain usable expressions for the phase difference by calculating the eigenvalues of $\Delta$ for that specific orientation and inserting them into eq 24. The stretching of polymer sheets, however, will give rise to molecular orientation parallel to the surface and therefore give rise to either $A, B$, or $C$ being collinear with ND. The orientation angles, $\theta, \phi$, and $\psi$, need to be measured to determine which situation, eq 27, 28, or 29, is the case.

A3. Measurement Of Optical Orienation. Determining whether the specimen is optically uniaxial or biaxial will assist in the measurement of optical orientation. If the isogyres are present in the interference figure, then the shape of the isogyres and the number of melatopes will reveal whether the specimen is uniaxial or biaxial, where two melatopes clearly signifies the material is biaxial. For only slightly biaxial materials, the two melatopes may not be easily distinguishable. The reader is directed to consult standard optical 


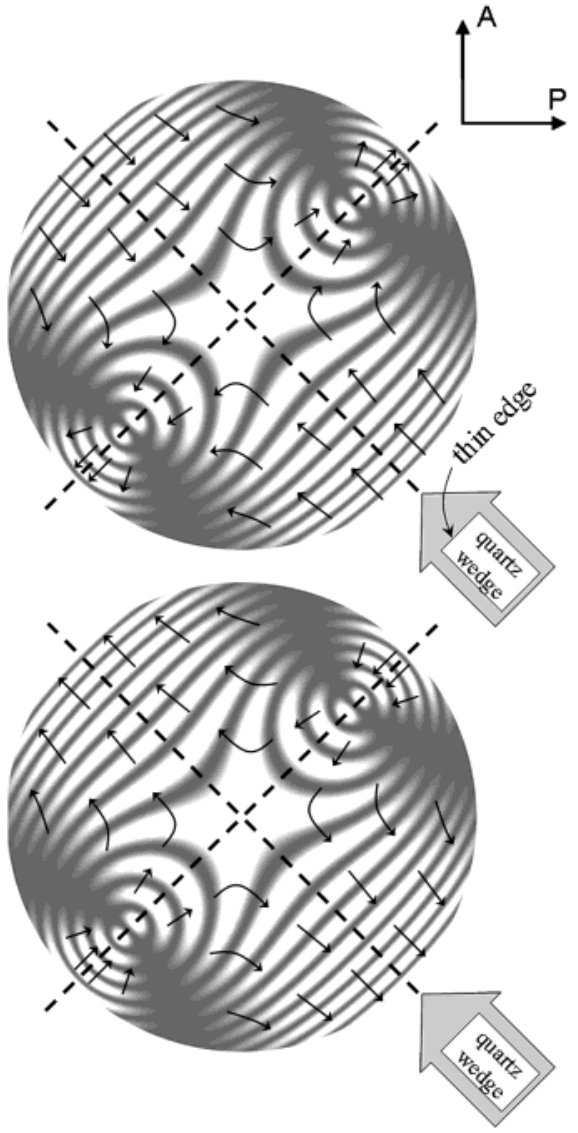

Figure 14. Determination of optical sign of bisectrix figure through the insertion of a quartz wedge into the optical path. The arrows indicate the direction of movement of the isochromates as a result of the insertion. Key: (a) positive (+); (b) negative (-) (figure adapted from ref 4).

crystallography texts ${ }^{4,5}$ for more information on the characteristics of isogyres. When the isogyres are not visible it may not be easy to a priori determine whether the material is biaxial or uniaxial, in which case the more general situation of biaxiallity should be assumed.

The measurement of the uniaxial orientation angles, $\theta$ and $\phi$, has al ready been studied in detail.5,6 For biaxial materials with either $A, B$, or $C$ normal to the surface, as the case with eqs $27-29$, it is a relatively straightforward procedure to determine the orientation, with the angle $\phi$ simply given by the observed rotation of the interference figure relative to MD. For other orientations analogous techniques to those used with uniaxial specimens may employed. However, as mentioned in the previous section this will usually not be expected with stretched polymer films, so focus will be placed on distinguishing between cases where a principal axis of the refractive index ellipsoid is collinear with ND.

For both images in Figure 14, the acute bisectrix is normal to the surface. To ascertain whether $A$ or $C$ is the acute bisectrix the optical sign of the material must be determined. This is done by inserting a quartz wedge microscope accessory into the optical path and observing the change on the position of the isochromates according to F igure 14. As explained earlier in the paper, this step is not necessary if the intrinsic refractive indices of the polymer chain are known.

\section{References and Notes}

(1) Kanai, T.; Cambell, G. A. Film Processing; Baker, W., Ed.; Carl Hanser Verlag: Munich, Germany, 1999.

(2) Osaki, S.; Uranishi, K. Polymer 1990, 31, 33-35.

(3) Sakamoto, K. J apan. J . Polym. Sci. Technol. 1991, 48, 671678.

(4) Bloss, F. D. An Introduction to the Methods of Optical Crystallography; Holt, Rinehart \& Wilson: New York, 1961.

(5) Wahlstrom, E. E. Optical Crystallography, 4th ed.; J ohn Wiley \& Sons: New York, 1969.

(6) Van Horn, B. L.; Winter, H. H. Appl. Opt. 2001, 40, 20892094.

(7) Wright, F. The Methods of Petrographic-Microscopic Research; Carnegie Inst.: Washington, DC, 1911.

(8) Galay, J.; Cakmak, M. J. Polym. Sci., Part B: Polym. Phys. 2001, 39, 1107-1121.

(9) Cakmak, M.; White, J . L.; Spruiell, J . E. Polym. Eng. Sci. 1989, 29, 1534-1543.

(10) Fouda, I. M.; Kabeel, M. A.; El-Sharkawy, F. M. J . Appl. Polym. Sci. 1998, 69, 33-44.

(11) Gohil, R. M. J . Appl. Polym. Sci. 1993, 48, 1635-1648.

(12) Werner, E.; J anocha, A.; Hopper, M. J .; Mackenzic, K.J . In Polyesters, Films, 2nd ed.; Mark, H. F., Bikales, N. M., Overberger, C. G., Menges, G., Kroschwitz, J . I., Eds.; J ohn Wiley \& Sons: New York, 1988; Vol. 12.

(13) Wlochowicz, A.; Rabiej, S.; J anicki, J . J . Appl. Polym. Sci. 1983, 28, 1335-1346.

(14) Kim, J. C.; Cakmak, M.; Zhou, X. Polymer 1998, 39, 42255234.

(15) Whitney, L. R. Toy mirror with transmissiveimagemode: US, 1999.

(16) Yamada, T.; Chisato, N. J . Appl. Polym. Sci. 1993, 48, 13991408.

(17) Tsunashima, K.; Toyoda, K.; Yoshii, T. Stretching Conditions Orientation, and Physical Properties of Biaxially Oriented Film; Kanai, T., Campbell, G. A., Eds.; Carl Hanser Verlag: Munich, Germany, 1999; pp 320-352.

(18) Yamada, T.; Nonomura, C. J . Appl. Polym. Sci. 1994, 52, 1393-1403.

(19) Born, M.; Wolf, E. Principles of Optics, 4th ed.; Pergamon Press: New York, 1969

(20) Huijts, R. A.; Peters, S. M. Polymer 1994, 35, 3119-3121.

(21) Samuels, R. J . Characterization of Deformation in Polycrystalline Polymer Films; Sweeting, O. J., Ed.; J ohn Wiley \& Sons: New York, 1968; Vol. 1, pp 255-364.

(22) Berreman, D. W. J . Opt. Soc. Am. 1973, 63, 1374-1380.

(23) Wöhler, H.; Haas, G.; Fritsch, M.; Mylnski, D. A. J . Opt. Soc. Am. 1988, 5, 1554-1557.

MA0347262 